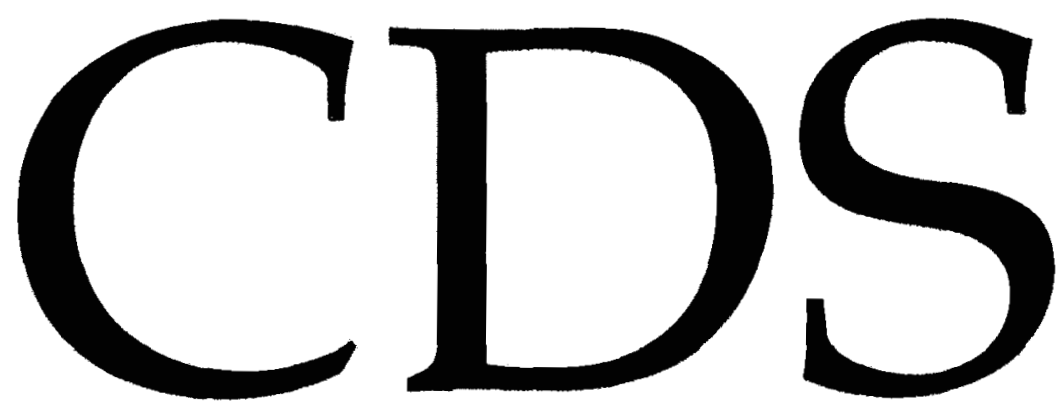

TECHNICAL MEMORANDUM NO. CIT-CDS 93-025

December 14, 1993

revised January 11, 1994

"Stability of Model Predictive Control with Soft Constraints"

A. Zheng and M. Morari

Control and Dynamical Systems

California Institute of Technology

Pasadena, CA 91125 


\title{
Stability of Model Predictive Control with Soft Constraints
}

\author{
Alex Zheng Manfred Morari * \\ Chemical Engineering, 210-41 \\ California Institute of Technology \\ Pasadena, CA 91125
}

April 26, 1994

\begin{abstract}
We derive stability conditions for Model Predictive Control (MPC) with hard constraints on the inputs and "soft" constraints on the ontputs for an infinitely long output horizon. We show that with state feedback MPC is globally asymptotically stabilizing if and only if all the eigenvalues of the open loop system are in the closed unit disk. With ontput feedback the eigenvalues must be strictly inside the unit circle. The on-line optimization problem defining MPC can be posed as a finite dimensional quadratic program even though the output constraints are specified over an infinite horizon.
\end{abstract}

\section{Introduction}

Many practical control problems are dominated by constraints. There are generally two types of constraints - input constraints and output constraints. The input constraints are always present and are imposed by physical limitations of the actuators which cannot be exceeded under any circumstances. Often, it is also desirable to keep specific outputs within certain limits for reasons related to plant operation, e.g. safety, material constraints, etc. It is usually unavoidable to exceed the output constraints, at least temporarily, for example, when the system is subjected to unexpected disturbances.

Industry has embraced Model Predictive Control (MPC), also referred to as moving horizon control and receding horizon control, as a powerful feedback strategy to control systems with constraints. The basic idea behind MPC is as follows: At sampling time $k, m$ future control moves are calculated such that an objective function over some (output) horizon is minimized subject to constraints. Only the first one of the $m$ computed control moves is implemented. At the next sampling time, the measurement is used to update the state estimate and the same calculations are repeated.

Rawlings and Muske [2] showed that global asymptotic stability of the constrained system can be guaranteed by making the horizon infinite, provided that the optimization problem defining the MPC controller is feasible. However, output constraints can lead to an infeasible optimization problem. They proposed to remove the output constraints during the initial portion of the infinite horizon to make the optimization problem feasible. Unfortunately, this can result in poor performance: the violation of the output constraints

*Author to whom all correspondence should be addressed: phone (818)395-4186, fax (818)568-8743, e-mail: MM@IMC.CALTECH.EDU 
during this initial portion of the infinite horizon can be very large in order to satisfy the constraints during the rest. Thus, large constraint violations may be experienced, when the computed control actions are implemented.

An alternative way to handle the feasibility problem is to relax the infeasible state constraints for the entire horizon and to penalize the extent of the violation. This technique is referred to as "constraint softening" [3]. The problem is that global stability may not be guaranteed. Zafiriou and Chiou [7] have derived some conditions for stability. However, these conditions are generally conservative and difficult to check.

In this note, we show that global asymptotic stability can be guaranteed for systems with mixed hard and soft constraints. Furthermore, in the case that the state must be estimated, we show that global stability is preserved by using an asymptotic observer. Finally, we show that the optimization can be cast as a finite dimensional quadratic program even though the output constraints are specified over the infinite horizon.

\section{State Feedback}

Consider the system

$$
\begin{aligned}
x(k+1) & =A x(k)+B u(k) \\
y(k) & =C x(k)
\end{aligned}
$$

where $x(k) \in \Re^{n_{x}}, u(k) \in \Re^{n_{n}}$ and $y(k) \in \Re^{n_{y}}$. Define the objective function as

$$
\Phi_{k}=\sum_{i=1}^{\infty} x(k+i \mid k)^{T} R x(k+i \mid k)+\sum_{i=0}^{m-1}\left[u(k+i \mid k)^{T} S u(k+i \mid k)+\Delta u(k+i \mid k)^{T} P \Delta u(k+i \mid k)\right]
$$

where $R>0, S>0, P \geq 0$, and $m$ is finite. $R, S$, and $P$ are symmetric. $(\cdot)(k+i \mid k)$ denotes the variable $(\cdot)$ a.t sampling time $k+i$ predicted at sampling time $k$. The control actions are generated by Controller MPC which is defined as follows.

Definition 1 Controller MPC: At sampling time $k$, the control move u(k) equals the first element $u(k \mid k)$ of the sequence $\{u(k \mid k), u(k+1 \mid k), \cdots, u(k+m-1 \mid k)\}$ which is the minimizer of the optimization problem

$$
\begin{aligned}
& J_{k}=\min _{\epsilon(k), u(k \mid k), \cdots, u(k+m-1 \mid k)} \Phi_{k}+\epsilon(k)^{T} Q \epsilon(k) \\
& \text { subject to }\left\{\begin{array}{cl}
|\Delta u(k+i \mid k)| \leq \Delta u^{\max } & i=0,1, \cdots, m-1 \\
u^{m i n} \leq u(k+i \mid k) \leq u^{\max } & i=0,1, \cdots, m-1 \\
\Delta u(k+i \mid k)=0 & i=m, m+1, \cdots, \infty \\
G x(k+i \mid k) \leq g+\epsilon(k) & i=0,1, \cdots, \infty \\
\epsilon(k) \geq 0
\end{array}\right.
\end{aligned}
$$

where $G \in \Re^{n_{G} \times n_{x}}$ and $Q>0$ is diagonal.

The input constraints represent physical limitations on the actuators which cannot be violated. The output constraints are softened by the slack variables $\epsilon(k)$. They can be violated temporarily, if necessary. In the long term, the penalty term $\epsilon(k)^{T} Q \epsilon(k)$ in the objective function will drive the slack variables to zero. The optimization problem (3) can be cast as a quadratic program.

The control problem is to bring the state to the origin. To make it well posed, the feasible region for

$$
\left\{\begin{array}{cl}
|\Delta u(k+i \mid k)| \leq \Delta u^{\text {max }} & i=0,1, \cdots, m-1 \\
u^{\text {min }} \leq u(k+i \mid k) \leq u^{\text {max }} & i=0,1, \cdots, m-1
\end{array}\right.
$$


must contain $u(k+i \mid k)=0, i=0,1, \cdots, m-1$, as an interior point. The feasible reagion for

$$
\left\{\begin{array}{c}
G x(k+i \mid k) \leq g+\epsilon(k) \quad i=0,1, \cdots, \infty \\
\epsilon(k)=0
\end{array}\right.
$$

contains $x(k+i \mid k)=0, i=0,1, \cdots, \infty$, as an interior point. Note that this implies $g>0$. Then we have the following theorem which extends the results in [2] for $\epsilon(k)=0 \forall k \geq 0$.

Theorem 1 The closed-loop system with Controller MPC is globally asymptotically stable if and only if the optimization problem (3) is feasible.

Proof: If the optimization problem is not feasible, the controller is not defined. Feasibility of the optimization problem implies that $J_{1}$ is finite. At sampling time $k+1$, let

$$
\left\{\begin{array}{l}
u^{*}(k+i \mid k+1)=u(k+i \mid k) \quad i=1,2, \cdots, m \\
\epsilon^{*}(k+1)=\epsilon(k)
\end{array}\right.
$$

Thus, $\left(u^{*}, \epsilon^{*}\right)$ is a feasible solution but may not be optimal. We have

$$
J_{k+1} \leq J_{k}-x(k+1)^{T} R x(k+1)-u(k)^{T} S u(k)-\Delta u(k)^{T} P \Delta u(k)
$$

which yields

$$
J_{k+1}+\sum_{i=1}^{k}\left[x(i+1)^{T} R x(i+1)+u(i)^{T} S u(i)+\Delta u(k)^{T} P \Delta u(k)\right] \leq J_{1}<\infty
$$

Note that we replaced $x(k+1 \mid k)$ with $x(k+1)$ since $x(k+1)=x(k+1 \mid k)$. This together with $R, S>0$ implies that $x(k) \rightarrow 0$ and $u(k) \rightarrow 0$ as $k \rightarrow \infty$.

Remark 1 This theorem also holds if $S \geq 0$ provided that at steady state $x=0$ if and only if $u=0$. By allowing $S=0$, we can introduce integral control to obtain offset-free tracking.

Remark 2 If $Q=\infty$, then the output constraints become hard and the optimization problem may not be feasible.

The following theorem states that for $Q<\infty$ feasibility of the optimization problem (3) is guaranteed for stable systems.

Theorem 2 If $A$ is stable, i.e. all eigenvalues of $A$ are strictly inside the unit circle, then the optimization problem. (3) is feasible $\forall m \geq 1$ and $\forall Q<\infty$.

Proof: All we have to do is to prove the feasibility of the optimization problem at the first sampling time. We will prove this theorem by construction. Since $A$ is stable, $x(k)$ is bounded $\forall k \geq 0$ for any initial condition. Then

$$
\left\{\begin{array}{l}
u^{*}(i \mid 1)=0 \\
\epsilon^{*}(1)=\max _{i \geq 1}|G x(i \mid 1)|_{\infty}<\infty
\end{array} \quad i=1,2, \cdots, m\right.
$$

satisfies all the constraints and results in $J_{1}<\infty$. Thus it is a feasible solution.

In light of results by Tsirukis and Morari [5], Balakrishnan et al [1], and Zheng and Morari [8], we can show that Theorem 2 also holds for stabilizable systems with poles in the closed unit disk provided that $m$ is sufficiently large. This is stated in the following theorem. 
Theorem 3 Assume that $\{A, B\}$ is stabilizable and that all eigenvalues of $A$ are in the closed unit disk. Then for a sufficiently large but finite value of $m$ the optimization problem (3) is feasible $\forall Q<\infty$.

Proof: See, for example, [5].

We have shown, that with $m$ properly chosen, Controller MPC globally asymptotically stabilizes any constrained stabilizable system with poles in the closed unit disk, using state feedback. When the inputs are constrained, i.e. $u^{\min } \leq u(k) \leq u^{\max } \forall k$, Sontag [4] showed that there does not exist a controller that globally stabilizes any system with poles outside the unit circle. ${ }^{1}$ Thus, the MPC controller globally stabilizes all constrained systems for which a global stabilization is possible.

Remark 3 Theorems 1, 2 and 3 hold as well if other norms for softening the output constraints are used.

\section{Output Feedback}

In the previous section, we assumed that the state is measured. Since the closed loop system may be nonlinear because of the constraints, we cannot apply the Separation Principle to prove stability for the output feedback case. Denote the state (output) at sampling time $k+i$ estimated at sampling time $k$ by $\hat{x}(k+i \mid k)(\hat{y}(k+i \mid k))$. We replace the state $x$ in the objective function (2) by its estimate $\hat{x}$. The state is estimated as follows.

$$
\begin{aligned}
\hat{x}(k \mid k) & =A \hat{x}(k-1 \mid k-1)+B u(k-1)+L(y(k)-\hat{y}(k \mid k-1)) \\
\hat{x}(k+i \mid k) & =A x(k+i-1 \mid k)+B u(k+i-1) \quad i \geq 1
\end{aligned}
$$

where $L$ is the observer gain. Combining this equation with equation (1) yields

$$
e(k+1)=(I-L C) A e(k)
$$

where $e(k)=x(k)-x(k \mid k)$. Thus equation (4) can be written as

$$
\begin{aligned}
\hat{x}(k \mid k) & =\hat{x}(k \mid k-1)+\operatorname{LCAe}(k-1) \\
\hat{x}(k+i \mid k) & =A \hat{x}(k+i-1 \mid k)+B u(k+i-1) \quad i \geq 1
\end{aligned}
$$

which yields

$$
\begin{aligned}
\xi(k \mid k) & =\operatorname{LCAe}(k-1) \\
\xi(k+i \mid k) & =A \xi(k+i-1 \mid k) \quad i \geq 1
\end{aligned}
$$

where $\xi(k+i \mid k)=\hat{x}(k+i \mid k)-\hat{x}(k+i \mid k-1)$. Then we have the following lemma.

Lemma 1 Assume that $A$ and $(I-L C) A$ are stable, i.e. all the eigenvalues are strictly inside the unit circle. Define

$$
\eta(k)=\max _{i \geq k}\left|G A^{i-k} e(i)\right|_{\infty}\left[\begin{array}{c}
1 \\
\vdots \\
1
\end{array}\right] \in \Re^{n_{G}}
$$

Then

$$
\begin{gathered}
\sum_{k=1}^{\infty} \sqrt{\sum_{i=0}^{\infty} \xi(k+i \mid k)^{T} R \xi(k+i \mid k)}<\infty \\
\sum_{k=1}^{\infty} \sqrt{\eta(k)^{T} Q \eta(k)}<\infty \\
\sum_{k=1}^{\infty}\left(\sqrt{\sum_{i=0}^{\infty} \xi(k+i \mid k)^{T} R \xi(k+i \mid k)}+\sqrt{\eta(k)^{T} Q \eta(k)}\right)^{2}<\infty
\end{gathered}
$$

\footnotetext{
${ }^{1}$ With constraints on $\Delta u$, we can also show that there does not exist a controller which globally stabilizes an unstable system.
} 
where $0<R, Q<\infty$.

Proof: From equations (5) and (7), we have

$$
|e(k)|_{2} \leq c_{1} k^{\alpha_{1}-1} \rho_{1}^{k}|e(0)|_{2}
$$

and

$$
|\xi(k+i \mid k)|_{2} \leq c_{2} i^{\alpha_{2}-1} \rho_{2}^{i}|\xi(k \mid k)|_{2} \leq c_{1} c_{2} i^{\alpha_{2}-1} k^{\alpha_{1}-1} \rho_{2}^{i} \rho_{1}^{k}|e(0)|_{2}
$$

where $\rho_{1}=\lambda_{\max }((I-L C) A)$ and $\rho_{2}=\lambda_{\max }(A) ; c_{1}$ and $c_{2}$ are constant; $\alpha_{1}$ and $\alpha_{2}$ are the multiplicies associated with the largest eigenvalues ${ }^{2}$ of $(I-L C) A$ and $A$, respectively. Here $\lambda_{\text {max }}(A)$ denotes the spectral radius of $A$. Stability of $A$ and $(I-L C) A$ implies that $\rho_{1}, \rho_{2}<1$. Thus,

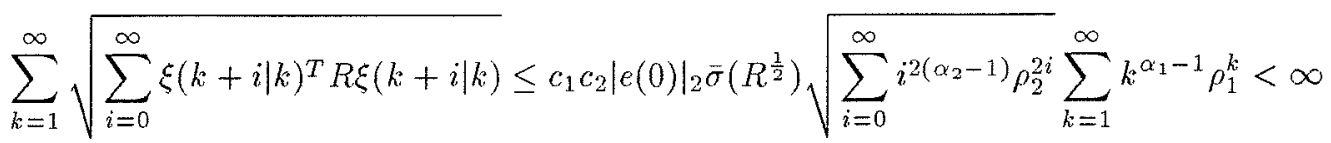

The other two expressions can be proven similarly.

Remark 4 If $A$ is unstable or has poles on the unit circle, Lemma 1 clearly does not hold.

The following theorem states that global asymptotic stability with output feedback can be guaranteed for stable systems.

Theorem 4 Assume that $A$ and $(I-L C) A$ are stable, i.e. all eigenvalues of $A$ and $(I-L C) A$ are strictly inside the unit circle. Then the overall system with Controller MPC and observer (A) is globally asymptotically stable.

Proof: Denote the weighted 2-norm $\sqrt{x^{T} R x}$ by $|x|_{R 2}$. Let

$$
\left\{\begin{array}{l}
u^{*}(k+i \mid k+1)=u(k+i \mid k) \quad i=1,2, \cdots, m \\
\epsilon^{*}(k+1)=\epsilon(k)+\eta(k)
\end{array}\right.
$$

Thus, $\left(u^{*}, \epsilon^{*}\right)$ is a feasible solution but may not be optimal. Define

$$
\begin{gathered}
U=\sum_{i=1}^{m}\left[|u(k+i \mid k)|_{S 2}^{2}+|\Delta u(k+i \mid k)|_{P 2}^{2}\right] \\
\alpha(k)=|\hat{x}(k+1 \mid k)|_{R 2}^{2}+|u(k)|_{S 2}^{2}+|\Delta u(k)|_{P 2}^{2}
\end{gathered}
$$

We have

$$
\begin{aligned}
J_{k+1} & \leq \sum_{i=2}^{\infty}|\hat{x}(k+i \mid k+1)|_{R 2}^{2}+U+\left|\epsilon^{*}(k+1)\right|_{Q 2}^{2} \\
& =\sum_{i=2}^{\infty}|\hat{x}(k+i \mid k)+\xi(k+i \mid k+1)|_{R 2}^{2}+U+|\epsilon(k)+\eta(k)|_{Q 2}^{2} \\
& \leq\left(\sqrt{\sum_{i=2}^{\infty}|\hat{x}(k+i \mid k)|_{R 2}^{2}+U+|\epsilon(k)|_{Q 2}^{2}}+\sqrt{\left.\sum_{i=2}^{\infty}|\xi(k+i \mid k+1)|_{R 2}^{2}+|\eta(k)|_{Q 2}\right)^{2}}\right. \\
& =\left(\sqrt{J_{k}-\alpha(k)}+\sqrt{\sum_{i=2}^{\infty}|\xi(k+i \mid k+1)|_{R 2}^{2}}+|\eta(k)|_{Q 2}\right)^{2}
\end{aligned}
$$

\footnotetext{
${ }^{2}$ The largest eigenvalue is defined to be the eigenvalue with the largest absolute value.
} 
Taking square root both sides yields

$$
\begin{aligned}
\sqrt{J_{k+1}} & \leq \sqrt{J_{k}-\alpha(k)}+\sqrt{\sum_{i=2}^{\infty}|\xi(k+i \mid k+1)|_{R 2}^{2}}+|\eta(k)|_{Q 2} \\
& \leq \sqrt{J_{k}}+\sqrt{\sum_{i=2}^{\infty}|\xi(k+i \mid k+1)|_{R 2}^{2}}+|\eta(k)|_{Q 2}
\end{aligned}
$$

which in turn yields

$$
\sqrt{J_{k+1}} \leq \sqrt{J_{1}}+\sum_{j=1}^{k}\left[\sqrt{\sum_{i=2}^{\infty}|\xi(j+i \mid j+1)|_{R 2}^{2}}+|\eta(j)|_{Q 2}\right]
$$

By Lemma 1, the second term on the right-hand-side is bounded for all $k$. Therefore, we have

$$
J_{k} \leq J^{\max }<\infty \quad \forall k>0
$$

From before, we have

$$
\begin{aligned}
& J_{k+1} \leq\left(\sqrt{J_{k}-\alpha(k)}+\sqrt{\sum_{i=2}^{\infty}|\xi(k+i \mid k+1)|_{R 2}^{2}}+|\eta(k)|_{Q 2}\right)^{2} \\
& =J_{k}-\alpha(k)+\left(\sqrt{\sum_{i=2}^{\infty}|\xi(k+i \mid k+1)|_{R 2}^{2}}+|\eta(k)|_{Q 2}\right)^{2}+2 \sqrt{J_{k}-\alpha(k)}\left(\sqrt{\sum_{i=2}^{\infty}|\xi(k+i \mid k+1)|_{R 2}^{2}}+|\eta(k)|_{Q 2}\right) \\
& \leq J_{k}-\alpha(k)+\left(\sqrt{\left.\sum_{i=2}^{\infty}|\xi(k+i \mid k+1)|_{R 2}^{2}+|\eta(k)|_{Q 2}\right)^{2}+2 \sqrt{J^{\text {max }}}}\left(\sqrt{\sum_{i=2}^{\infty}|\xi(k+i \mid k+1)|_{R 2}^{2}}+|\eta(k)|_{Q 2}\right)\right.
\end{aligned}
$$

which yields

$$
J_{k+1}+\sum_{i=1}^{k} \alpha(i) \leq J_{1}+\sum_{i=1}^{k}\left[\left(\sqrt{\sum_{i=2}^{\infty}|\xi(k+i \mid k+1)|_{R 2}^{2}}+|\eta(k)|_{Q 2}\right)^{2}+2 \sqrt{J^{m a x}}\left(\sqrt{\sum_{i=2}^{\infty}|\xi(k+i \mid k+1)|_{R 2}^{2}}+|\eta(k)|_{Q 2}\right)\right]
$$

By Lemma 1 and boundness of $J^{\max }$, the second term is bounded for all $k$. Thus,

$$
J_{k+1}+\sum_{i=1}^{k} \alpha(i)=J_{k}+\sum_{i=1}^{k}\left[|\hat{x}(i+1 \mid i)|_{R 2}^{2}+|u(i)|_{S 2}^{2}+|\Delta u(i)|_{P 2}^{2}\right]<\infty
$$

Following a similar argument as in the proof of Theorem 1, we can therefore conclude that $x(k) \rightarrow 0$ and $u(k) \rightarrow 0$ as $k \rightarrow \infty$.

The following theorem shows that the output constraints over the infinite horizon can be replaced by the output constraints over the finite horizon. A similar result was derived by Rawlings and Muske [2].

Theorem 5 Assume that $A$ is stable. Given any $\hat{x}(k \mid k)$ and $\epsilon(k) \geq 0$, there exists a finite $N$ such that

$$
G \hat{x}(k+i \mid k) \leq g+\epsilon(k) \quad \forall i \geq N
$$


Proof: We need only prove this theorem for $\epsilon(k)=0$ : since $\epsilon(k) \geq 0 \forall k, G \hat{x}(k+i \mid k) \leq g \forall i \geq N$ implies $G \hat{x}(k+i \mid k) \leq g+\epsilon(k) \forall i \geq N$. WLOG, assume that $A$ is nonsingular. ${ }^{3}$ Consider a zero input, i.e. $u(k+i \mid k)=0, i=0, \cdots, m-1$, and denote the value of the objective function for this input sequence by $J_{k}^{*}$. Then,

$$
J_{k} \leq J_{k}^{*}=\hat{x}(k \mid k)^{T} \sum_{i=1}^{\infty}\left(A^{T}\right)^{i} R A^{i} \hat{x}(k \mid k) \equiv \hat{x}(k \mid k)^{T} \Pi \hat{x}(k \mid k)
$$

where $\Pi$ is positive definite and bounded since $A$ is nonsingular and stable. Also we have

$$
\begin{aligned}
J_{k} & =\sum_{i=1}^{\infty} \hat{x}(k+i \mid k)^{T} R \hat{x}(k+i \mid k)+\sum_{i=0}^{m-1}\left[u(k+i \mid k)^{T} S u(k+i \mid k)+\Delta u(k+i \mid k)^{T} P \Delta u(k+i \mid k)\right]+\epsilon(k)^{T} Q \epsilon(k) \\
& \geq \sum_{i=m}^{\infty} \hat{x}(k+i \mid k)^{T} R \hat{x}(k+i \mid k) \\
& =\hat{x}(k+m \mid k)^{T} \Pi \hat{x}(k+m \mid k)
\end{aligned}
$$

Combining these two inequalities, we obtain

$$
\hat{x}(k+m \mid k)^{T} \Pi \hat{x}(k+m \mid k) \leq \hat{x}(k \mid k)^{T} \Pi \hat{x}(k \mid k)
$$

which yields

$$
|\hat{x}(k+m \mid k)|_{2} \leq \kappa(\Pi)|\hat{x}(k \mid k)|_{2}
$$

where $\kappa(\Pi)<\infty$ denotes the condition number of $\Pi$. Finally,

$$
\begin{aligned}
|G \hat{x}(k+m+N \mid k)|_{\infty} & =\left|G A^{N} \hat{x}(k+m \mid k)\right|_{\infty} \\
& \leq\left|G A^{N} \hat{x}(k+m \mid k)\right|_{2} \\
& \leq \bar{\sigma}(G) \bar{\sigma}\left(A^{N}\right)|\hat{x}(k+m \mid k)|_{2} \\
& \leq \bar{\sigma}(G) \bar{\sigma}\left(A^{N}\right) \kappa(\Pi)|\hat{x}(k \mid k)|_{2}
\end{aligned}
$$

where $\bar{\sigma}(G)$ denotes the largest singular value of $G$. If $N$ is such that

$$
\bar{\sigma}(G) \bar{\sigma}\left(A^{N+i}\right) \kappa(\Pi)|\hat{x}(k \mid k)|_{2} \leq \min _{j}\left(g_{j}\right) \quad \forall i \geq 0
$$

then

$$
G \hat{x}(k+i \mid k) \leq g \quad \forall i \geq N+m
$$

$\min _{j}\left(g_{j}\right)>0$ and stability of $A$ imply that a finite $N$ exists.

\section{Example}

Consider the system

$$
\begin{aligned}
x(k+1) & =\left[\begin{array}{cc}
0.655 & -0.1673 \\
0.1673 & 0.9825
\end{array}\right] x(k)+\left[\begin{array}{l}
0.1637 \\
0.0175
\end{array}\right] u(k) \\
y(k) & =\left[\begin{array}{c}
-2 \\
1
\end{array}\right] x(k)
\end{aligned}
$$

\footnotetext{
${ }^{3}$ If $A$ is singular, we can write $A=T^{-1}\left[\begin{array}{cc}\Sigma_{1} & 0 \\ 0 & \Sigma_{2}\end{array}\right] T$ where $\Sigma_{1}>0$ and $\Sigma_{2}$ is nilpotent. Define $\tilde{x}(k)=T x(k)$ and $\left[\begin{array}{l}\tilde{x}_{1}(k+1) \\ \tilde{x}_{2}(k+1)\end{array}\right]=\left[\begin{array}{cc}\Sigma_{1} & 0 \\ 0 & \Sigma_{2}\end{array}\right]\left[\begin{array}{c}\tilde{x}_{1}(k) \\ \tilde{x}_{2}(k)\end{array}\right]$. Then, after a finite number of sampling times, $\tilde{x}_{2}$ becomes identically zero since $\Sigma_{2}$ is nilpotent. Thus it suffices to consider the reduced system with $\tilde{x}_{1}$ as its states.
} 
which is obtained from the continuous-time transfer function $\frac{-2 s+1}{(s+1)^{2}}$ with a sampling time of 0.2 . The initial condition is $x(0)=\left[\begin{array}{ll}1.5 & 1.5\end{array}\right]^{T}$. The output is constrained between \pm 1 . Since the system exhibits inverse response behavior, hard output constraints can cause stability problems ([6]). To use the approach proposed in [2], the output constraint at the first sampling time must be ignored to make the optimization problem feasible. We can also use the approach presented in this note and soften the output constraints over the infinite horizon. The following parameter values are used:

$$
p=\infty, m=5, R=\left[\begin{array}{cc}
5 & -2 \\
-2 & 2
\end{array}\right], S=0, P=0, Q=I
$$

Using the arguments leading to Theorem 5 one can show that the state constraints will be satisfied automatically after 35 time steps. Thus, the output constraints must be enforced only over a finite horizon of length 35. The responses for the two approaches are depicted in Figure 1. A very large overshoot is observed for the controller designed via the approach proposed in [2].

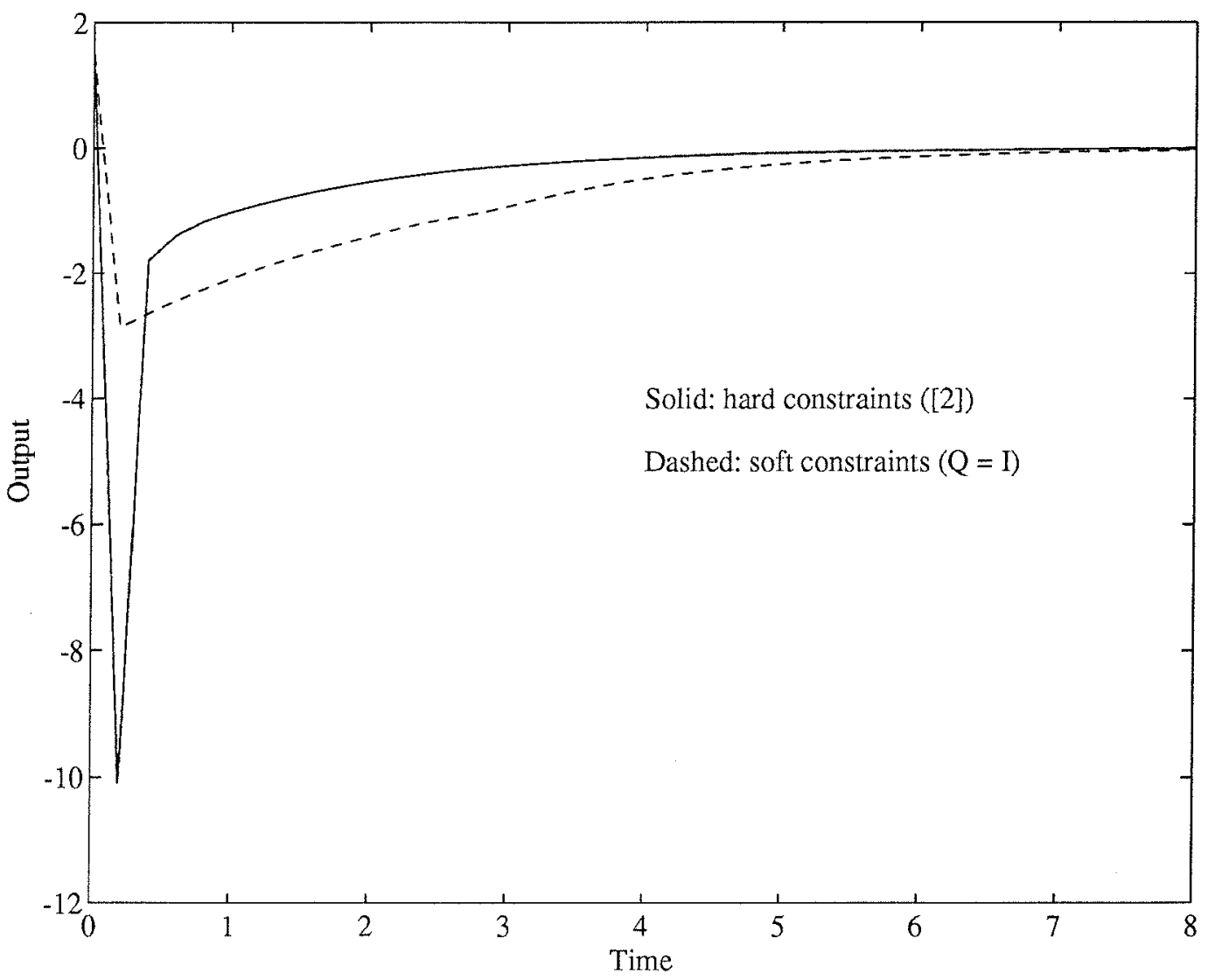

\section{Conclusions}

We analyzed the closed loop stability for an infinite horizon MPC algorithm with soft and hard constraints. We showed that global stability can guaranteed for both state feedback and output feedback. The on-line optimization problem can be cast as a finite dimensional quadratic program. 


\section{References}

[1] V. Balakrishnan, A. Zheng, and M. Morari. Constrained stabilization of discrete-time linear systems. In Proc. Workshop on Advances in Model-Based Predictive Control, University of Oxford, 1993. Oxford University Press.

[2] J. Rawlings and K. R. Muske. The stability of constrained receding horizon control. IEEE Transactions on Automatic Control, 38:1512-1516, 1993.

[3] N. L. Ricker, T. Subrahmanian, and T. Sim. Case studies of model-predictive control in pulp and paper production. In T. J. McAvoy and E. Zafiriou, editors, Model Based Process Control - Proc. of the 1988 IFAC Workshop, Oxford, 1989. Pergamon Press.

[4] E. Sontag. An algebraic approach to bounded controllability of linear systems. International Journal of Control, 39:181-188, 1984.

[5] A. Tsirukis and M. Morari. Controller design with actuator constraints. In Conf. on Decision and Control, pages 2623-2628, 1992.

[6] E. Zafiriou. Robust model predictive control of processes with hard constraints. Comp. Chem. Engng., 14(4/5):359-371, 1990.

[7] E. Zafiriou and H.-W. Chiou. Output constraint softening for SISO model predictive control. Proceedings of American Control Conf., pages 372-276, 1993.

[8] A. Zheng and M. Morari. Global stabilization of linear discrete-time systems with bounded controls a model predictive control approach. Proceedings of American Control Conf., 1994. Submitted. 